\title{
Stability of bicontinuous cubic phases in ternary amphiphilic systems with spontaneous curvature
}

\author{
U. S. Schwarz \\ Max-Planck-Institut für Kolloid und Grenzflächenforschung, Am Mühlenberg, Haus 2, 14476 Golm, \\ Germany and Department of Materials and Interfaces, The Weizmann Institute of Science, \\ Rehovot 76100, Israel \\ G. Gompper \\ Max-Planck-Institut für Kolloid und Grenzflächenforschung, Am Mühlenberg, Haus 2, 14476 Golm, \\ Germany and Institut für Festkörperforschung, Forschungszentrum Jülich, 52425 Jülich, Germany
}

(Received 8 March 1999; accepted 10 November 1999)

\begin{abstract}
We study the phase behavior of ternary amphiphilic systems in the framework of a curvature model with nonvanishing spontaneous curvature. The amphiphilic monolayers can arrange in different ways to form micellar, hexagonal, lamellar, and various bicontinuous cubic phases. For the latter case we consider both single structures (one monolayer) and double structures (two monolayers). Their interfaces are modeled by the triply periodic surfaces of constant mean curvature of the families G, D, P, C(P), I-WP, and F-RD. The stability of the different bicontinuous cubic phases can be explained by the way in which their universal geometrical properties conspire with the concentration constraints. For vanishing saddle-splay modulus $\bar{\kappa}$, almost every phase considered has some region of stability in the Gibbs triangle. Although bicontinuous cubic phases are suppressed by sufficiently negative values of the saddle-splay modulus $\bar{\kappa}$, we find that they can exist for considerably lower values than obtained previously. The most stable bicontinuous cubic phases with decreasing $\bar{\kappa}<0$ are the single and double gyroid structures since they combine favorable topological properties with extreme volume fractions. (C) 2000 American Institute of Physics.
\end{abstract} [S0021-9606(00)70306-0]

\section{INTRODUCTION}

As a function of concentrations and temperature, amphiphilic systems generically form many different phases, each of which corresponds to a specific geometrical arrangement of the amphiphilic interfaces. ${ }^{1,2}$ Most ternary amphiphilic systems feature the disordered micellar, the hexagonal and the lamellar phase. Here the amphiphilic monolayers, which separate regions of water from regions of oil, form spheres, cylinders and lamellae, respectively. However, many of these systems also have stable cubic phases. ${ }^{3-5}$ Near the disordered micellar phase one often finds a micellar cubic phase and near the lamellar phase a bicontinuous cubic phase. In the micellar cubic phase, the interfaces form spheres as they do in the disordered micellar phase, only that now they are packed in an orderly fashion. In the bicontinuous cubic phase, they form sheets which span the whole sample in all directions of space. ${ }^{6,7}$ Each of these triply periodic surfaces has a cubic Bravais lattice and divides space into two unconnected but intertwined labyrinths filled with water and oil, respectively.

In this work, the polymorphism of ternary amphiphilic systems is studied in the framework of a curvature model with nonvanishing spontaneous curvature. We assume that the shape of the amphiphilic monolayers is determined by their bending rigidity even in the presence of concentration constraints. Therefore we model them by surfaces of constant mean curvature. This encompasses the spheres, cylinders, and lamellae of the noncubic phases; for the bicontinu- ous cubic phases, the surfaces of constant mean curvature are triply periodic. Safran and co-workers considered the same geometries and showed that the interplay between the bending energy and the volume constraints can explain some aspects of the generic phase behavior of ternary amphiphilic systems. ${ }^{8-10}$ In particular, Wang and Safran ${ }^{10}$ considered the single structure (one monolayer) and the double structure (two monolayers) from the $D$-family. Their calculation relied on data for triply periodic surfaces of constant mean curvature, which were found numerically by Anderson, who investigated the geometrical properties of the $\mathrm{D}, \mathrm{P}, \mathrm{C}(\mathrm{P})$, I-WP, and F-RD families. ${ }^{11,12}$ However, the bicontinuous cubic phases most often identified experimentally are the various gyroid structures which correspond to the G-family. Data for the G-family were calculated only recently by Große-Brauckmann. ${ }^{13,14}$ In this work, we consider as bicontinuous cubic phases single and double structures for all families for which the required geometrical data are known, including the G-family. Indeed, we find that for negative values of the saddle-splay modulus $\bar{\kappa}$, of all bicontinuous cubic phases considered it is mainly the gyroid structures which are stable. They can exist for values of $\bar{\kappa}$ which are twice as negative as the values for which the structures from the D-family have been found to be stable previously. ${ }^{10} \mathrm{We}$ will show that this finding can be explained nicely by considering the interplay between certain universal geometrical properties of the various bicontinuous cubic phases and the volume constraints within the Gibbs triangle. In fact we find 
that the gyroid structures are so favorable because they have exceptional topological properties and at the same time can accommodate extreme volume fractions of oil and water.

\section{CURVATURE MODEL AND NONCUBIC PHASES}

The elastic properties of amphiphilic interfaces are described by the Canham-Helfrich expression for the elastic energy per unit area, ${ }^{15,16}$

$$
f_{\text {elastic }}=2 \kappa\left(H-c_{0}\right)^{2}+\bar{\kappa} K,
$$

where $H=\left(c_{1}+c_{2}\right) / 2$ and $K=c_{1} c_{2}$ are mean and Gaussian curvatures, respectively, and $c_{1}$ and $c_{2}$ the two principal curvatures of the surface. The two elastic moduli are the bending rigidity $\kappa$ and the saddle-splay modulus $\bar{\kappa}$. The main temperature dependence is carried by the spontaneous curvature $c_{0}$. For systems with water, oil and nonionic surfactants $C_{i} E_{j}$, it is found experimentally that $c_{0} \propto\left(T_{b}-T\right)$, where $T_{b}$ is the balanced temperature. ${ }^{17}$ Here positive curvature is defined to be curvature towards the oil regions, thus oil is the interior phase below the balanced temperature and water above. For example, $c_{0} \approx 1 /(6 l)$ and $c_{0} \approx 1 /(12 l)$ for $\mathrm{H}_{2} \mathrm{O} / \mathrm{C}_{14} / \mathrm{C}_{12} \mathrm{E}_{5}$ at $T=20^{\circ} \mathrm{C}$ and $T=34{ }^{\circ} \mathrm{C}$, respectively, where $l \approx 1.5 \mathrm{~nm}$ is the amphiphile length in the monolayer and $T_{b}=48^{\circ} \mathrm{C} .{ }^{17}$ In this work, we assume a positive value for the spontaneous curvature, as it is typical for surfactant systems below the balanced temperature, so that oil is the interior phase. The tendency to bend towards the oil regions decreases with temperature since the headgroups' hydration decreases. For the case of a negative spontaneous curvature, water and oil have to be interchanged in the various structures as well as in the phase diagrams presented below. This is usually the case not only for surfactants above the balanced temperature, but also for lipid systems where the monolayers tend to bend towards the water regions due to their bulky tail regions.

We consider the dimensionless free energy per unit volume,

$$
f=\frac{1}{2 \kappa c_{0}^{3} V} \int d A f_{\text {elastic }}=\frac{A}{c_{0} V}\left(\frac{H}{c_{0}}-1\right)^{2}-\frac{2 \pi \chi r}{c_{0}^{3} V},
$$

where the integration extends over the neutral surface $A$ of the amphiphilic monolayers, $V$ is the overall volume, and $r$ $=-\bar{\kappa} / 2 \kappa$. For the first (bending) term in Eq. (1), the area integration can be carried out since we only consider surfaces of constant mean curvature. For the second (topological) term, we employ the Gauss-Bonnet theorem, $\int d A K$ $=2 \pi \chi$, where $\chi$ is the Euler characteristic of the surface. The curvature model is only stable for $-2 \kappa \leqslant \bar{\kappa} \leqslant 0$ or 0 $\leqslant r \leqslant 1 .{ }^{18}$ Experimentally there is no straightforward way to measure $\bar{\kappa}$, but usually a small negative value is assumed.

The phase diagram at constant temperature is a function of the volume fractions $\rho_{W}, \rho_{O}$ and $\rho_{A}$ of water, oil, and amphiphile, respectively, which are restricted to the Gibbs triangle by $\rho_{W}+\rho_{O}+\rho_{A}=1$. We consider amphiphiles of length $l$, which have a tail length $\alpha l$ and a head size (1 $-\alpha) l$, with $0<\alpha<1$. Throughout this paper we will use $\alpha$ $=1 / 2$, i.e., we will consider amphiphiles which have head and tail regions of similar size, as they are for $\mathrm{C}_{12} \mathrm{E}_{5}$. The hydrocarbon volume fraction is given by $\mathrm{v}=\rho_{O}+\alpha \rho_{A} .{ }^{9} \mathrm{In}$ order to parametrize concentration space, it is convenient to use $v$ and the ratio $w=\rho_{A} /\left[\left(\rho_{O}+\alpha \rho_{A}\right) c_{0} l\right]$. The amphiphile volume fraction $\rho_{A}$ is taken to be $A l / V$. If the amphiphile is assumed to occupy the space between the two parallel surfaces at distances $\alpha l$ and $(1-\alpha) l$ from the neutral surface, this is a very accurate approximation when the amphiphile length $l$ is small compared to the extension of the amphiphilic aggregates, and gives reasonable results even for the extreme case of oil-free spherical micelles for $\alpha \simeq 1 / 2$.

In the following all lengths are measured in units of $l$. The free-energy densities of the noncubic phases follow from Eq. (2) as

$$
\begin{aligned}
& f_{L}(w, \mathrm{v})=w \mathrm{v}, \\
& f_{C}(w, \mathrm{v})=w \mathrm{v}\left(\frac{w}{4}-1\right)^{2}, \\
& f_{S}(w, \mathrm{v}, r)=w \mathrm{v}\left[\left(\frac{w}{3}-1\right)^{2}-\frac{r w^{2}}{9}\right] .
\end{aligned}
$$

Note that only $f_{S}$ depends on $r$, since the other two structures have no Gaussian curvature. Since we only consider the freeenergy contribution due to the curvature elasticity of the amphiphilic monolayers, for the noncubic phases $f$ has a trivial $\mathrm{V}$-dependence. However, due to close packing, spheres and cylinders have maximal volume fractions $\mathrm{v}_{\max }=\sqrt{2} \pi / 6$ $=0.74$ and $\pi / 2 \sqrt{3}=0.91$, respectively. With $w, \mathrm{v}$, and $r$, the model has a three-dimensional parameter space.

It can be seen from Eq. (3) that the Maxwell construction is not possible for this model, since $f_{L}$ is not concave. Therefore we use the intersections of the free-energy densities of different phases as an indication for the location of phase transitions. We want to remark parenthetically that Wang and Safran ${ }^{10}$ considered the free energy per unit area (rather than per unit volume); this amounts to an overall factor of $w \mathrm{~V} / c_{0}$ (the dimensionless specific area) in the freeenergy density of all phases. As long as the location of the phase transitions is estimated from the intersection of the free-energy curves, the two approaches are equivalent. However, for a calculation of two-phase regions, the use of the free energy per unit volume becomes essential-since in general coexisting phases will have different amphiphile concentrations.

For $r=0$ (vanishing saddle-splay modulus) and $\mathrm{v}$ $<0.74$ (no excluded volume effects), Eqs. (3), (4), and (5) imply the phase sequence $L \rightarrow C \rightarrow S$ with decreasing $w . f_{S}$ begins to rise again for $w \leqslant 3$. This is identified with the emulsification failure, the coexistence of $S$ and an excess oil phase at low amphiphile concentration. ${ }^{10}$ In this paper, we define the emulsification failure not as the minimum in $f_{S}$ in regard to $w$, but by using a Maxwell construction in regard to $w$ between $S$ and an excess oil phase, i.e., by solving $\partial f_{S} / \partial w=f_{S} / w$ for $w$. With increasing $r$ (and $\mathbf{v}<0.74$ ), spheres become more favorable, while cylinders are increasingly suppressed and finally disappear for $r>1 / 4$. In particular, the phase boundaries $S-C, S-L, C-L$ and the emul- 


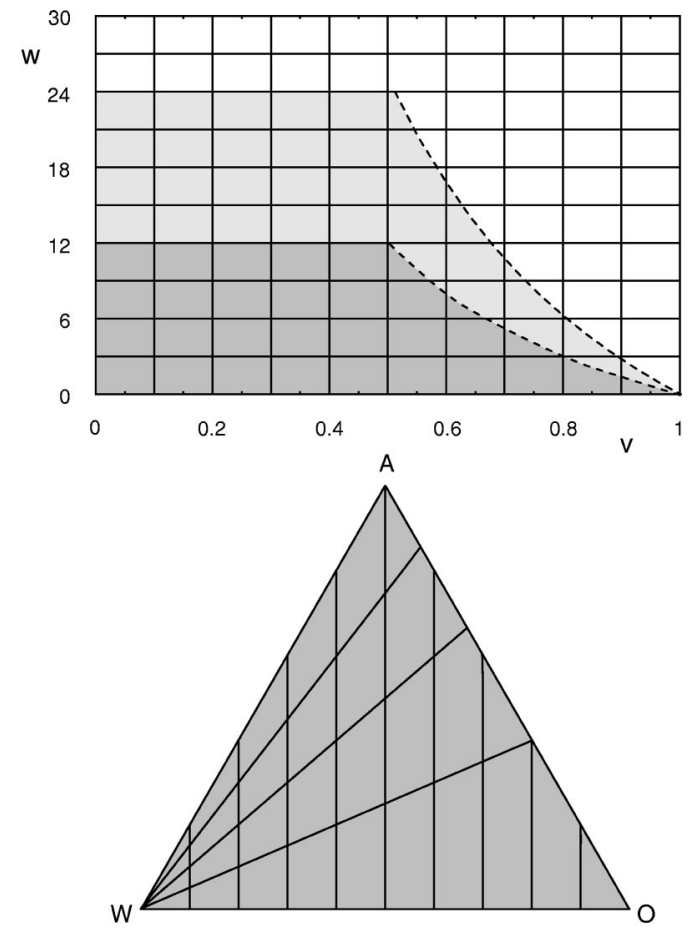

FIG. 1. Correspondence between the $(\mathrm{V}, w)$-plane and the Gibbs triangle. Only a certain part of the $(\mathrm{v}, w)$-plane is mapped onto the Gibbs triangle. The mapping depends on the values for the amphiphile chain length $\alpha$ and for the spontaneous curvature $c_{0}$. Throughout this work we use $\alpha=1 / 2$ (length is measured in units of amphiphile length). For $c_{0}=1 / 6$ and $c_{0}$ $=1 / 12$, the darkly and lightly shaded parts are mapped onto the Gibbs triangle, respectively. Water, amphiphile, and oil apex of the Gibbs triangle are denoted by $W, A$, and $O$, respectively.

sification failure are obtained from Eqs. (3), (4), and (5) to be $w=24 /(7-16 r), w=6 /(1-r), w=8$, and $w=3 /(r-1)$, respectively.

It is important to realize that not all values of $(\mathrm{v}, w)$ are physically relevant. In Fig. 1 we show the mapping between the $(\mathrm{v}, w)$-plane and the Gibbs triangle for ternary mixtures. The binary limits $O-W, W-A$, and $A-O$ of the Gibbs triangle correspond to $\rho_{O}+\rho_{W}=1, \rho_{W}+\rho_{A}=1$ and $\rho_{A}+\rho_{O}$ $=1$, respectively. In the $(\mathrm{V}, w)$-plane, this corresponds to the lines $w=0, w=1 /\left(\alpha c_{0}\right)$ and $w=(1 / v-1) /\left((1-\alpha) c_{0}\right)$, respectively. The line $V=0$ is mapped onto the $W$-apex. Figure 1 demonstrates (for $\alpha=1 / 2$ ) this mapping of the ( $\mathrm{V}, w)$-plane onto the Gibbs triangle for $c_{0}=1 / 6$ and $c_{0}=1 / 12$. As mentioned above, these values correspond to the system $\mathrm{H}_{2} \mathrm{O} / \mathrm{C}_{14} / \mathrm{C}_{12} \mathrm{E}_{5}$ at $T=20^{\circ} \mathrm{C}$ and $T=34{ }^{\circ} \mathrm{C}$, respectively. ${ }^{17}$ Smaller/larger values for the spontaneous curvature (which corresponds to higher/lower temperatures or other components) increases/decreases the size of the relevant region in Fig. 1(a) to larger/smaller values of $w$. One also can see in Fig. 1(b) that for $\alpha=1 / 2$ lines of constant $v$ are perpendicular to the $W-O$ side and lines of constant $w$ are straight lines through the $W$-apex.

\section{BICONTINUOUS CUBIC PHASES}

\section{A. Properties of constant-mean-curvature surfaces}

We model the amphiphilic monolayers in the bicontinuous cubic phases by triply periodic surfaces of constant mean

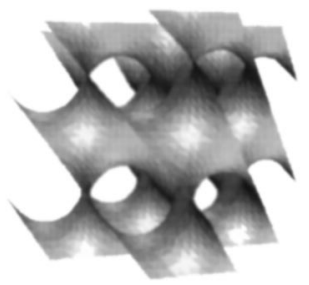

(a)

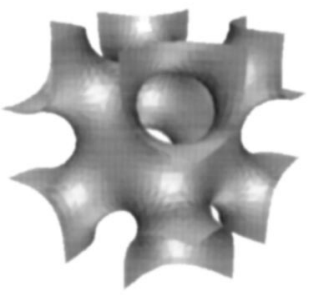

(c)

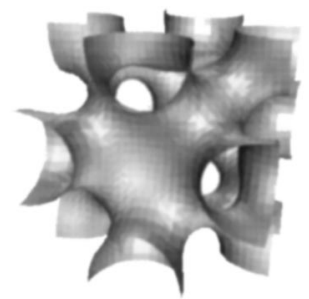

(b)

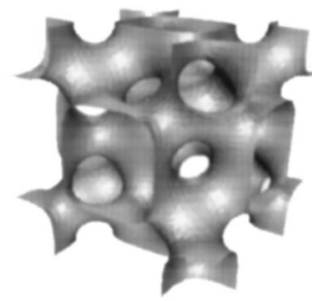

(d)
FIG. 2. Minimal surface members for the following families of triply periodic surfaces of constant mean curvature: (a) D, (b) C(P), (c) I-WP, and (d) F-RD. Shown is one conventional unit cell. For single structures with vanishing mean curvature, these surfaces represent the oil-water interfaces.

curvature $H=\left(c_{1}+c_{2}\right) / 2$. For the special case $H=0$, this leads to triply periodic minimal surfaces with $c_{1}=-c_{2}$ and $K=c_{1} c_{2} \leqslant 0$. Due to the Gauss-Bonnet theorem, $\int d A K$ $=2 \pi \chi$, these surfaces have a negative Euler characteristic $\chi$ per unit cell. Triply periodic minimal surfaces are commonly used to model all kinds of extended sheetlike structures in condensed matter systems, in particular the midsurfaces of the lipid bilayers in inverse cubic phases which are very prominent in lipid-water mixtures. ${ }^{6,7}$ Before 1970 only three cubic triply periodic minimal surfaces have been known [D, $\mathrm{P}$ and $\mathrm{C}(\mathrm{P})]{ }^{19,20}$ Then Schoen described five more [G, F-RD, I-WP, O, C-TO and C(D) ]. ${ }^{21}$ Today some more examples are known, ${ }^{22,23}$ but none of them seems to be of physical relevance. Karcher proved in 1989 not only the existence of the triply periodic minimal surfaces described by Schoen, but also that the simpler of them can be deformed into triply periodic surfaces of constant (nonzero) mean curvature. ${ }^{24} \mathrm{As}$ for any triply periodic surface, space is divided into two percolating labyrinths. A shift of $H$ to positive/negative values shrinks/expands one labyrinth, while it expands/shrinks the other. Thus two branches are generated, which both end in cubic arrangements of (infinitesimally connected and possibly self-intersecting) spheres. Since the Euler characteristic is a topological quantity connected to the genus $g$ of the surface by $\chi=2(1-g)$, it does not change within a family. Anderson studied the cubic families D, P, C(P), I-WP, and F-RD and calculated for a conventional unit cell of unit lattice constant both the volume fraction $v$ of one labyrinth as a function of scaled mean curvature $H^{*}$ (the volume fraction of the other labyrinth follows as $1-\mathrm{v}$ ) and the scaled surface area $A^{*}$ as a function of $\mathrm{v} \cdot{ }^{11,12}$ Recently $\mathrm{v}\left(H^{*}\right)$ and $A^{*}(\mathrm{v})$ were calculated also for the G-family by Große-Brauckmann. ${ }^{13,14}$

In this work we consider the families $\mathrm{G}, \mathrm{D}, \mathrm{P}, \mathrm{C}(\mathrm{P})$, I-WP, and F-RD, for which these geometrical data are avail- 

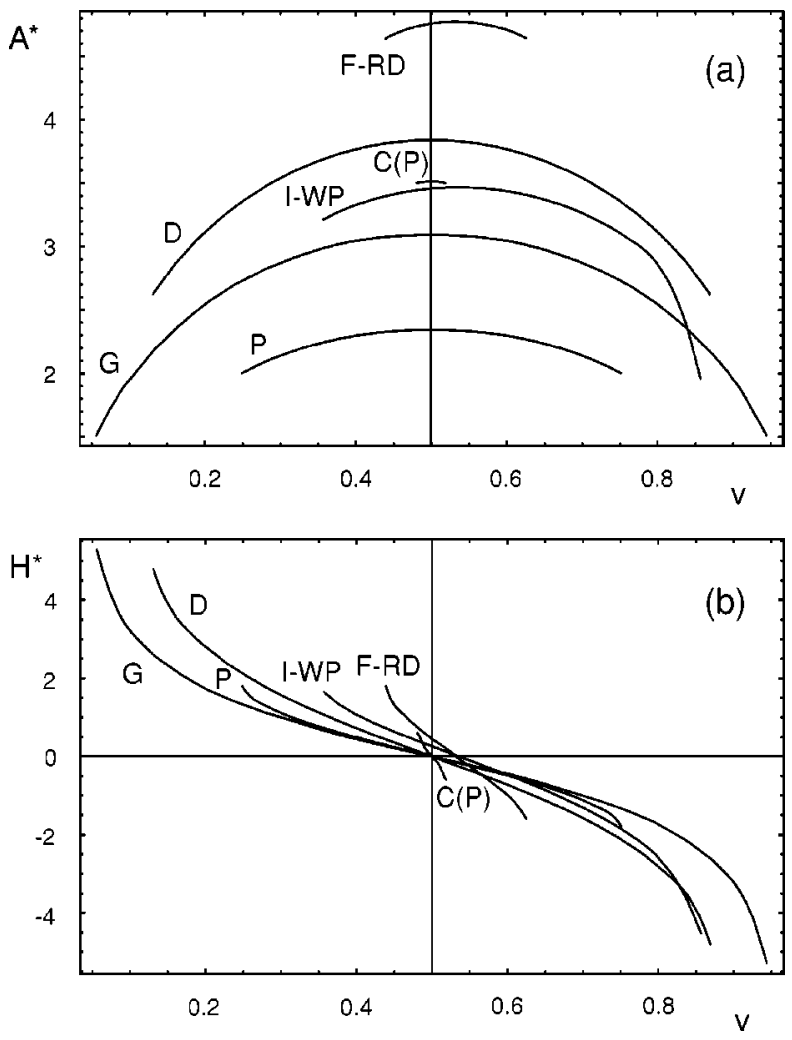

FIG. 3. Geometrical data for triply periodic surfaces of constant mean curvature: (a) scaled surface area $A^{*}$ and (b) scaled mean curvature $H^{*}$ as a function of volume fraction $v$. Each family exists only for a certain $\mathrm{V}$-interval. We only show the two branches connected by the minimal surface member; there exist other branches within the same $\mathrm{V}$-intervals which correspond to dense arrangements of nearly spherical regions connected by small necks. They are assumed to have no physical relevance. The two branches used are symmetrical for $\mathrm{G}, \mathrm{D}, \mathrm{P}$, and $\mathrm{C}(\mathrm{P})$ since their minimal surface members are balanced.

able. Their minimal surface members are shown in Fig. 2 for $\mathrm{D}, \mathrm{C}(\mathrm{P}), \mathrm{I}-\mathrm{WP}$, and F-RD in one conventional unit cell. The numerically calculated data points for $\mathrm{V}\left(H^{*}\right)$ and $A^{*}(\mathrm{~V})$ per conventional unit cell are taken from Refs. 11, 13; rearrangement and interpolation with cubic splines provides smooth functions $\mathrm{v}\left(H^{*}\right), H^{*}(\mathrm{v})$, and $A^{*}(\mathrm{v})$. The data are used only up to the extremal values of $\mathrm{v}$, beyond which the curves bend backwards. Beyond these points, the surfaces resemble ensembles of spheroidal regions connected by nearly unduloidal necks, which we do not consider to be of physical relevance. ${ }^{25}$ The functions $H^{*}(\mathrm{v})$ and $A^{*}(\mathrm{v})$ as used in this work are plotted in Fig. 3 for the six families considered. In fact, only one of the two curves for each family carries independent information since the other one can be constructed by using $d A^{*}=2 H^{*} d \mathrm{v} .{ }^{12}$ Each family only exists over a certain range of volume fractions; the extreme cases are the G-family with $v \in[0.056,0.944]$ and the $C(P)$-family with $\mathrm{V} \in[0.481,0.519]$. If the minimal surface member of a family divides space into two congruent labyrinths, they have the same volume fraction $v_{0}=1 / 2$, the two branches are symmetric to each other and the minimal surface as well as the family itself is called balanced. For the structures considered here, this is the case for $\mathrm{G}, \mathrm{D}, \mathrm{P}$, and $\mathrm{C}(\mathrm{P})$. For these structures, the curves $H^{*}(\mathrm{~V})$ and $A^{*}(\mathrm{~V})$ are symmetrical with respect to $v=1 / 2$. For the nonbalanced families I-WP and
TABLE I. Properties of the families of triply periodic surfaces of constant mean curvature, which are related to their minimal surface members. $\chi$ and $A_{0}$ are the Euler characteristic and the surface area in the conventional unit cell [the value of $A_{0}$ is known exactly in terms of elliptic functions for $\mathrm{G}, \mathrm{D}$, P, C(P), and I-WP (Refs. 21, 48)]. $\Gamma_{0}=\left(A_{0}^{3} / 2 \pi|\chi|\right)^{1 / 2}$ is the topology index; the structures are ordered with respect to decreasing magnitude of $\Gamma_{0} \cdot \mathrm{v}_{0}$ is the volume fraction of one of the two labyrinths; the volume fraction of the other one follows as $1-\mathrm{v}_{0} \cdot c=d \mathrm{v}\left(H^{*}\right) /\left.d H^{*}\right|_{H^{*}=0}$, where $\mathrm{v}\left(H^{*}\right)$ is the volume fraction of one of the two labyrinths for the corresponding family of surfaces of constant mean curvature. The values for D, I-WP, and P are taken from Ref. 12, the ones for G, F-RD, and $\mathrm{C}(\mathrm{P})$ are obtained from our spline interpolation of the numerical data of Ref. 12. $c^{\prime}=-A_{0}^{2} / 2 \pi \chi$ is the estimate of $c$ as derived in the text. Note that $c A_{0}$ gives a very similar hierarchy as $c^{\prime} A_{0}=\Gamma_{0}^{2}$.

\begin{tabular}{lrcccccc}
\hline \hline & $\chi$ & $A_{0}$ & $\Gamma_{0}$ & $\mathrm{v}_{0}$ & $c$ & $c^{\prime}$ & $c A_{0}$ \\
\hline $\mathrm{G}$ & -8 & 3.0914 & 0.7667 & 0.5 & 0.2191 & 0.1901 & 0.6773 \\
$\mathrm{D}$ & -16 & 3.8378 & 0.7498 & 0.5 & 0.1411 & 0.1465 & 0.5415 \\
$\mathrm{I}-\mathrm{WP}$ & -12 & 3.4641 & 0.7425 & 0.536 & 0.1385 & 0.1592 & 0.4798 \\
$\mathrm{P}$ & -4 & 2.3451 & 0.7163 & 0.5 & 0.2117 & 0.2188 & 0.4965 \\
$\mathrm{~F}-\mathrm{RD}$ & -40 & 4.7707 & 0.6573 & 0.532 & 0.0665 & 0.0906 & 0.3173 \\
$\mathrm{C}(\mathrm{P})$ & -16 & 3.5105 & 0.6560 & 0.5 & 0.0466 & 0.1226 & 0.1636 \\
\hline \hline
\end{tabular}

F-RD, the two labyrinths are of different topology and $\mathrm{v}_{0}$ $\neq 1 / 2$. In Table I, we collect data connected to the minimal surface members of each family, which we will use later in our discussion.

In the vicinity of the minimal surface, the volume fraction $\vee$ as a function of scaled mean curvature $H^{*}$ can be approximated as $\mathrm{\vee}\left(H^{*}\right)=\mathrm{v}_{0}-c H^{*}+\mathcal{O}\left(H^{* 2}\right)$. With $d A^{*}$ $=2 H^{*} d \mathrm{v}$ it follows that

$$
\begin{aligned}
& H^{*}(\mathrm{v})=-\frac{\left(\mathrm{v}-\mathrm{v}_{0}\right)}{c}+\mathcal{O}\left(\left(\mathrm{v}-\mathrm{v}_{0}\right)^{2}\right), \\
& A^{*}(\mathrm{v})=A_{0}-\frac{\left(\mathrm{v}-\mathrm{v}_{0}\right)^{2}}{c}+\mathcal{O}\left(\left(\mathrm{v}-\mathrm{v}_{0}\right)^{3}\right)
\end{aligned}
$$

near the minimal surface. The values for $A_{0}, \mathrm{v}_{0}, c$ are given in Table I. Since the surfaces of constant mean curvature can be expected to have similar geometrical properties as parallel surfaces in the vicinity of a minimal surface, the magnitude of $c$ can be estimated as follows. If $t$ denotes the perpendicular distance from the minimal to its parallel surface, to lowest order in $t$ the volume fraction $\mathrm{v}$ and the mean curvature $H^{*}$, averaged over the surface in the whole unit cell, are given by $\mathrm{v}=\mathrm{v}_{0}+A_{0} t$ and $H^{*}=2 \pi \chi t / A_{0}$, respectively. ${ }^{26,27}$ Thus $H^{*}$ $=2 \pi \chi\left(\mathrm{v}-\mathrm{v}_{0}\right) / A_{0}^{2}$ and $c=-A_{0}^{2} / 2 \pi \chi$ for the parallel surface case. The corresponding numbers are given in Table I; except for $\mathrm{C}(\mathrm{P})$, the overall agreement with the numerical data for $c$ is remarkably good.

\section{B. Single and double structures}

The simplest case of a cubic bicontinuous phase in a ternary amphiphilic system is a single structure where the amphiphilic monolayers form one triply periodic surface. Then one labyrinth is filled with oil and the other with water. For balanced families, filling either of the two labyrinths with oil gives the same single structure; for the nonbalanced families I-WP and F-RD, this yields different single struc- 
TABLE II. Distribution of oil, water, and amphiphile in the different structural types of bicontinuous cubic phases. Q denotes single structures, $\mathrm{Q}_{\mathrm{I}}$ double structures of type I (oil-in-water), and $\mathrm{Q}_{\text {II }}$ double structures of type II (water-in-oil or inverse). Although double structures consist of two amphiphilic monolayers, for this classification we consider each structure to consist of one triply periodic minimal surface separating two percolating labyrinths.

\begin{tabular}{clll}
\hline \hline Structure & Labyrinth 1 & \multicolumn{1}{c}{ Surface } & Labyrinth 2 \\
\hline $\mathrm{Q}$ & water & monolayer & oil \\
$\mathrm{Q}_{\mathrm{I}}$ & oil & water-filled bilayer & oil \\
$\mathrm{Q}_{\mathrm{II}}$ & water & oil-filled bilayer & water \\
\hline \hline
\end{tabular}

tures which we denote by I, WP, F, and RD, respectively. ${ }^{25}$ Here the symbols I and F correspond to the curves plotted for I-WP and F-RD in Fig. 3. Since mean curvature is defined as curvature towards the oil regions, WP and RD follow by the replacements $\mathrm{V} \rightarrow 1-\mathrm{V}$ and $H \rightarrow-H$ from the data of $\mathrm{I}$ and $\mathrm{F}$ plotted in Fig. 3, respectively. Thus, altogether we consider 8 different single structures, which exist for the volume intervals $[0.056,0.944]$ for $\mathrm{G},[0.131,0.869]$ for $\mathrm{D},[0.249,0.751]$ for $\mathrm{P}, \quad[0.481,0.519]$ for $\mathrm{C}(\mathrm{P}),[0.357,0.857]$ for $\mathrm{I}$, $[0.143,0.643]$ for WP, $[0.439,0.625]$ for $\mathrm{F}$, and $[0.375,0.561]$ for RD.

To each single structure corresponds a double structure where the amphiphilic monolayers form two triply periodic surfaces arranged roughly parallel and on either side of the minimal surface of the corresponding single structure. Both surfaces divide space into two labyrinths which are topologically equivalent to those of the initial structure. However, since two amphiphilic monolayers are present, now these labyrinths are filled with the same component and separated by a bilayer which is filled with the other component. Single and double structures are also known as monolayer and bilayer structures. ${ }^{25,27} \mathrm{~A}$ double structure can be either of type I (water-filled bilayer) or of type II (oil-filled bilayer). ${ }^{28}$ There will be no problem below to distinguish the symbol I for double structure of type I from the symbol I for one of the two simple structures of the I-WP family. In Table II, we summarize the classification of bicontinuous cubic structures. In the following, single structures, type I double structures and type II double structures are abbreviated as $\mathrm{Q}, \mathrm{Q}_{\mathrm{I}}$, and $\mathrm{Q}_{\mathrm{II}}$, respectively. $\mathrm{Q}_{\mathrm{II}}$ structures are also known as inverse bicontinuous cubic phases. In Fig. 4 we show single

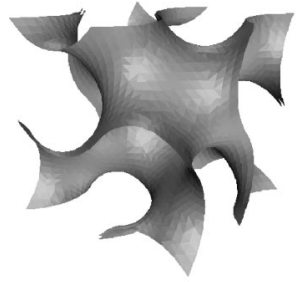

(a)

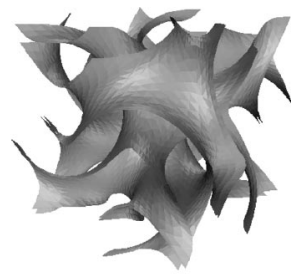

(b)
FIG. 4. (a) Single and (b) double structure of the G-family. The two gyroid structures are the most stable bicontinuous cubic phases for negative saddle splay modulus $\bar{\kappa}$. For the double gyroid of type I, the water forms the sheetlike region between the two monolayers which effectively form a bilayer in (b). For the double gyroid of type II, it fills the two channel networks which are separated by this bilayer. and double gyroid structures in one conventional unit cell. For many surfactant-water and lipid-water systems, $\mathrm{G}_{\text {II }}$ and/or $\mathrm{D}_{\mathrm{II}}$ are well established; moreover there are reports on $\mathrm{P}_{\mathrm{II}}$ and $\mathrm{G}_{\mathrm{I}}$ structures in these binary amphiphilic systems. ${ }^{3-5}$ Q structures in binary systems have been discussed only theoretically so far. ${ }^{29-31}$ For many systems with water, oil, and surfactant, stable bicontinuous cubic phases have been reported, however often without identification of their space group (see, e.g., Ref. 32 for the system $\mathrm{H}_{2} \mathrm{O} / \mathrm{C}_{10} / \mathrm{C}_{12} \mathrm{E}_{5}$ ). The best established identification is a number of $\mathrm{Q}_{\mathrm{II}}$ structures for the system DDAB-water-styrene. ${ }^{33}$ There are a few reports on $\mathrm{Q}$ structures, ${ }^{11,34}$ and none on $\mathrm{Q}_{\mathrm{I}}$ structures. However, several speculations on single structures can be found in the literature (e.g., in Refs. 27, 35), and our recent theoretical work on ternary systems with vanishing spontaneous curvature suggests that they should have some physical relevance. ${ }^{36}$ To our knowledge, hardly nothing is known on bicontinuous cubic phases in ternary systems with water, oil, and lipid.

In order to construct a double structure from a given family of triply periodic surfaces of constant mean curvature, we take two surfaces corresponding to $H$ and $-H$. Thus there is one $\mathrm{Q}_{\mathrm{I}}$ and one $\mathrm{Q}_{\mathrm{II}}$ structure for each of the six families. For $\mathrm{Q}_{\mathrm{I}}$ structures, the minimal surface case corresponds to $v=1$. As both surfaces accumulate mean curvature in their respective branches, the volume fraction decreases until the first labyrinth reaches its minimal size (which is the minimal volume fraction of the corresponding single structure). The volume intervals covered by the $\mathrm{Q}_{\mathrm{I}}$ structures turn out to be $[0.112,1.0]$ for $\mathrm{G}_{\mathrm{I}},[0.262,1.0]$ for $\mathrm{D}_{\mathrm{I}},[0.498,1.0]$ for $\mathrm{P}_{\mathrm{I}},[0.962,1.0]$ for $\mathrm{C}(\mathrm{P})_{\mathrm{I}},[0.624,1.0]$ for $\mathrm{I}^{-\mathrm{WP}_{\mathrm{I}} \text { and }}$ $[0.818,1.0]$ for $F-R_{I}$. The $\mathrm{Q}_{\mathrm{II}}$ structures follow from the corresponding $\mathrm{Q}_{\mathrm{I}}$ structures by an interchange of oil and water. Thus here the minimal surface case corresponds to $\mathrm{V}$ $=0$, and the volume fractions covered are complementary to the ones given for the $\mathrm{Q}_{\mathrm{I}}$ structures.

\section{Curvature and topology index}

For bicontinuous cubic structures, the free-energy density depends on the hydrocarbon volume fraction $\mathrm{v}$ in a nontrivial way, since only one or two amphiphilic aggregates are present. The lattice constant is denoted with $a$. For a given value of $\mathrm{v}$, the mean curvature $H(\mathrm{v}, a)=H^{*}(\mathrm{v}) / a$ and the surface area $A(\mathrm{~V}, a)=A^{*}(\mathrm{~V}) a^{2}$ within a unit cell are determined by the curves plotted in Fig. 3. The amphiphile concentration $\rho_{A}=A(\mathrm{~V}, a) / a^{3}=A^{*}(\mathrm{v}) / a$ fixes $a$, so that $a$ $=A^{*}(\mathrm{~V}) / \rho_{A}=A^{*}(\mathrm{~V}) /\left(w \vee c_{0}\right)$. Using the Gauss-Bonnet theorem for the Gaussian curvature term, we find from Eq. (2) for the free-energy density of a bicontinuous cubic structure,

$$
\begin{aligned}
f_{\mathrm{BC}}(w, \mathrm{v}, r) & =\frac{A}{c_{0} V}\left(\frac{H^{*}(\mathrm{v})}{c_{0} a}-1\right)^{2}-\frac{2 \pi \chi r}{\left(c_{0} a\right)^{3}} \\
& =w \mathrm{v}[\Lambda(\mathrm{v}) w \mathrm{v}-1]^{2}+r \frac{(w \mathrm{v})^{3}}{\Gamma(\mathrm{v})^{2}},
\end{aligned}
$$

with 


$$
\Lambda(\mathrm{v})=\frac{H^{*}(\mathrm{v})}{A^{*}(\mathrm{v})}, \quad \Gamma(\mathrm{v})=\left(\frac{A^{*}(\mathrm{v})^{3}}{2 \pi|\chi|}\right)^{1 / 2}
$$

Here $\chi$ denotes the Euler characteristic of the interfaces within one conventional unit cell, as given in Table I. The curvature index $\Lambda(\mathrm{v})$ and the topology index $\Gamma(\mathrm{v})$ are two universal geometrical quantities which characterize a surface in three-dimensional space. Their significance can be understood rather easily from the observation that both the mean and Gaussian curvatures can be made dimensionless by multiplying them with appropriate powers of $V / A$, which is the only relevant length scale; this implies $H(V / A)=\Lambda$ and $|K|(V / A)^{2}=2 \pi|\chi| V^{2} / A^{3}=1 / \Gamma^{2}$. It is not difficult to see that both quantities are not only invariant under scale transformations, but also under a change of unit cell. They also occur in integral geometry, a mathematical theory concerned with invariant geometrical measures. ${ }^{37}$ For convex bodies in threedimensional space there exist two independent isoperimetric inequalities and therefore two independent isoperimetric ratios, which usually are chosen to be $\Lambda$ and $1 / \Gamma^{2}$. The equivalence between these quantities and the isoperimetric ratios holds since for surfaces of constant mean curvature, the integral mean curvature, which is one of the Minkowski functionals of integral geometry, can be replaced by $H A$. The curvature index $\Lambda$ describes how strongly the structures is curved and the topology index $\Gamma$ describes its porosity (the larger its value, the less holes the structure has). For minimal surfaces, $\Lambda$ vanishes and $\Gamma$ remains the only relevant quantity. Its significance for amphiphilic systems has been pointed out by Hyde, ${ }^{27}$ and its variation as a function of crystallographic determinants has been studied recently by Fodgen and Hyde. ${ }^{38}$ For multicontinuous structures build from $n$ sheets, the curvature index is smaller and the topology index is larger by a factor of $n$ compared to the corresponding single structure. In particular, for double structures the curvature index is half and the topology index is twice as large as for single structures. Thus, double structures are less porous than single structures since they have disconnected surfaces. We can infer from Table I that the minimal gyroid is the least porous of the single structures with $\Gamma=0.7667$. We want to remark parenthetically that-surprisinglybicontinuous random surfaces have similar values of $\Gamma$ as single cubic structures. For example, it can be shown exactly that the isosurfaces of Gaussian random fields with $\langle H\rangle=0$ have $\Gamma=\sqrt{8} / \pi=0.9003 .{ }^{39,40}$ Since this value is only slightly larger than that of the single gyroid phase, it can be concluded that the random sponge phase on average features only few disconnected or multiple sheets. However, when the random sponge's interfaces are made to acquire curvature, the topology index grows strongly with $\Lambda$ since disconnected parts proliferate. ${ }^{40}$ The opposite is true for the families of surfaces of constant mean curvature considered here; if the mean curvature is increased from zero, the topology remains the same, but the area content decreases. Therefore the topology index decreases, since for given topology it measures the specific surface area.

In order to evaluate the free-energy density of the bicontinuous cubic phases from Eq. (7) as a function of $w, \mathrm{v}$, and $r$, curvature and topology index as defined in Eq. (8) have to
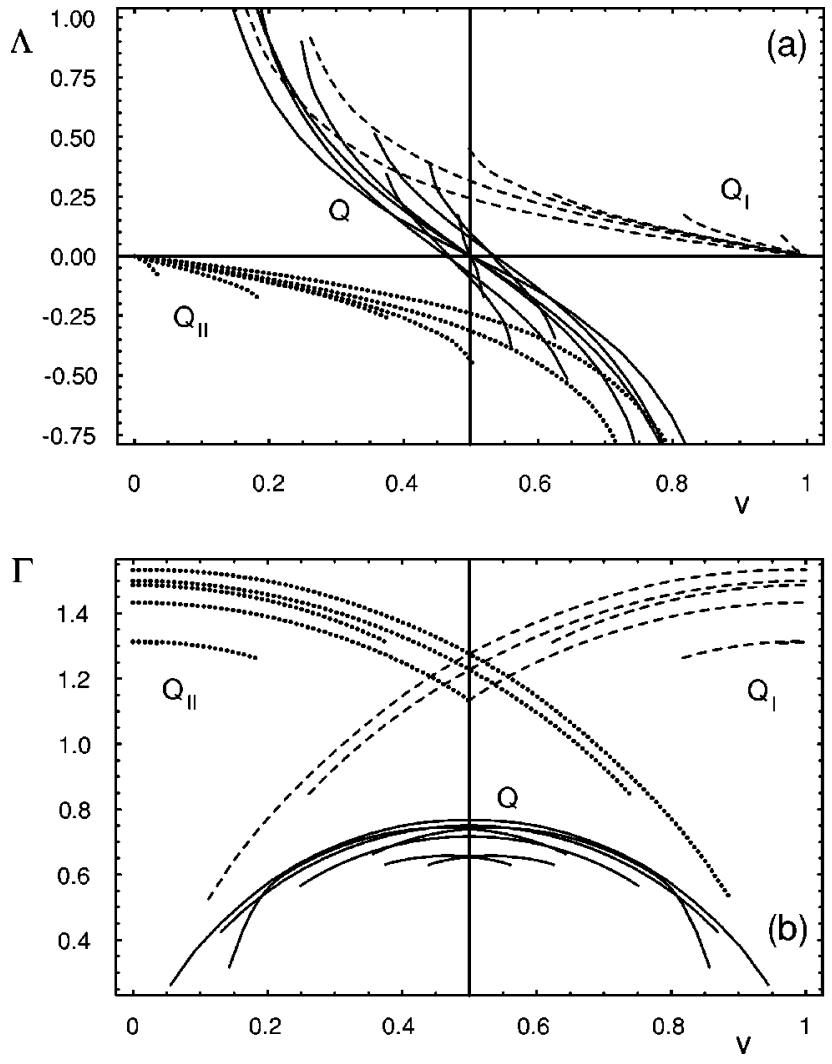

FIG. 5. (a) Curvature index $\Lambda$ and (b) topology index $\Gamma$ as a function of volume fraction $v$ for all structures considered. Although the different structures can be identified according to their $\mathrm{V}$-interval of existence (compare Fig. 3), here we intend to demonstrate only the difference between the three structural types. For $\mathrm{Q}$ (solid lines), $\mathrm{Q}_{\mathrm{I}}$ (dashed lines), and $\mathrm{Q}_{\mathrm{II}}$ structures (dotted lines) the curvature index vanishes and the topology index attains its maximal value for $v=v_{0}, v=1$, and $v=0$, respectively. $Q_{I I}$ structures and $\mathrm{Q}$ structures with $\mathbf{v}>\mathrm{V}_{0}$ have negative curvature indices and therefore are not stable for $c_{0}>0$.

be calculated as a function of $\mathrm{V}$. For the $8 \mathrm{Q}$ structures, they follow in a straightforward way from the data plotted in Fig. 3. In order to derive them for the $6 \mathrm{Q}_{\mathrm{I}}$ structures, we first consider the nonbalanced case, thus the two labyrinths 1 and 2 have different topologies (e.g., for I-WP, we have $1=\mathrm{I}$ and $2=\mathrm{WP})$. We first construct $\mathrm{V}_{\mathrm{I}}\left(H^{*}\right)=\mathrm{v}_{1}\left(H^{*}\right)+\mathrm{v}_{2}\left(H^{*}\right)$ and then invert it to obtain $H_{\mathrm{I}}^{*}(\mathrm{~V})$, the mean curvature of both surfaces as a function of the overall volume fraction (which is distributed onto both labyrinths). The surface area then follows as $A_{\mathrm{I}}^{*}(\mathrm{v})=A_{1}^{*}\left(\mathrm{v}_{1}\left(H_{\mathrm{I}}^{*}(\mathrm{v})\right)\right)+A_{2}^{*}\left(\mathrm{v}_{2}\left(H_{\mathrm{I}}^{*}(\mathrm{v})\right)\right)$. The Euler characteristic is $\chi_{\mathrm{I}}=\chi_{1}+\chi_{2}$ (with $\chi_{1}=\chi_{2}$ given in Table I). With $H_{\mathrm{I}}^{*}(\mathrm{v}), A_{\mathrm{I}}^{*}(\mathrm{v})$, and $\chi_{\mathrm{I}}$ calculated, we then can evaluate $\Lambda$ and $\Gamma$ in Eq. (8) for the nonbalanced $Q_{I}$ structures. For the nonbalanced $\mathrm{Q}_{\mathrm{II}}$ structures, we have to evaluate them using $H_{\mathrm{II}}^{*}(\mathrm{v})=-H_{\mathrm{I}}^{*}(1-\mathrm{v}), A_{\mathrm{II}}^{*}(\mathrm{v})=A_{\mathrm{I}}^{*}(1$ $-\mathrm{v})$, and $\chi_{\mathrm{II}}=\chi_{\mathrm{I}}$. For the balanced families, one has $\mathrm{v}_{1}$ $=\mathrm{v}_{2}=\mathrm{v} / 2, H_{\mathrm{I}}^{*}(\mathrm{v})=H^{*}(\mathrm{v} / 2), A_{\mathrm{I}}^{*}(\mathrm{v})=2 A^{*}(\mathrm{v} / 2), H_{\mathrm{II}}^{*}(\mathrm{v})$ $=-H^{*}((1-\mathrm{v}) / 2) \quad$ and $\quad A_{\text {II }}^{*}(\mathrm{v})=2 A^{*}((1-\mathrm{v}) / 2)$. This amounts to using $\Lambda_{\mathrm{I}}(\mathrm{v})=\Lambda(\mathrm{V} / 2) / 2, \quad \Gamma_{\mathrm{I}}(\mathrm{v})=2 \Gamma(\mathrm{v} / 2)$, $\Lambda_{\mathrm{II}}(\mathrm{v})=-\Lambda((1-\mathrm{v}) / 2) / 2$, and $\Gamma_{\mathrm{II}}(\mathrm{v})=2 \Gamma((1-\mathrm{v}) / 2)$ in Eq. (8). Note the minus sign for the curvature of the $\mathrm{Q}_{\text {II }}$ structures since the monolayers change their orientation compared to the $\mathrm{Q}_{\mathrm{I}}$ structures. In Fig. 5 we plot $\Lambda$ and $\Gamma$ as a function of $v$ for all single and double structures consid- 
ered. Recall that the minimal surface case corresponds to $\mathrm{V}$ $=\mathrm{V}_{0}, 1$ and 0 for $\mathrm{Q}, \mathrm{Q}_{\mathrm{I}}$, and $\mathrm{Q}_{\mathrm{II}}$ structures, respectively; in these cases, the curvature index disappears and the topology index acquires its maximum value. In Table I, we give the values of $\Gamma_{0}=\Gamma\left(\mathrm{V}=\mathrm{v}_{0}\right)$ for all families considered. This implies the hierarchy G, D, I-WP, P, C(P), F-RD with decreasing $\Gamma_{0}$. From Fig. 5 we also see that the single structures I and WP in fact can become better than single D for certain values of $\mathrm{V}$.

We see from Fig. 5 that single structures for $\mathrm{v}>\mathrm{v}_{0}$ and all $\mathrm{Q}_{\mathrm{II}}$ structures have negative values for the curvature index $\Lambda$. From Eq. (7) it therefore follows that these structures have $f_{\mathrm{BC}}>w \mathrm{~V}=f_{L}$, compare Eq. (3); thus they are always less stable than the lamellar phase. In other words, since we consider the case that the monolayers prefer to bend towards the oil regions (positive spontaneous curvature $c_{0}$ ), in the framework of the curvature model no phase can be stable which curves towards the water regions. One should note, however, that single structures for $\mathrm{V}>\mathrm{V}_{0}$ or $\mathrm{Q}_{\mathrm{II}}$ structures could occur even for positive $c_{0}$ if they were stabilized by other contributions to the free energy which are neglected in our treatment. For the case of negative $c_{0}$, the situation is reversed; now the monolayers prefer to bend towards the water regions, and from the double structures the (inverse) $\mathrm{Q}_{\mathrm{II}}$ and not the $\mathrm{Q}_{\mathrm{I}}$ structures are stable. In general, for ternary systems one expects $\mathrm{Q}$ in the middle of the Gibbs triangle (possibly in the vicinity of a microemulsion phase for surfactant systems), $Q_{I}$ near the binary side amphiphile-oil and $\mathrm{Q}_{\text {II }}$ near the binary side amphiphile-water. For surfactant systems, which usually have $c_{0}>0$ at room temperature, the curvature model predicts the stability of $Q$ and $Q_{I}$ structures; for lipid systems, which usually have $c_{0}<0$ at room temperature, $\mathrm{Q}$ and (inverse) $\mathrm{Q}_{\mathrm{II}}$ structures are predicted. This general prediction conforms with the predominance of inverse phases for lipid-water mixtures. Note that the surfactant systems DDAB-water-styrene ${ }^{33}$ mentioned above is an exception to our distinction between surfactant and lipid systems since the two tails result in $c_{0}<0$ and therefore lead to inverse phases like in the lipid case.

\section{PHASE BEHAVIOR}

\section{A. Phase diagrams for $r=0$}

Altogether we consider 17 different phases, 3 noncubic, 8 single, and 6 double structures of type I. Moreover there always exists an emulsification failure at low amphiphile-tooil ratios. We first discuss the case of vanishing saddle-splay modulus, $r=0$. For the bicontinuous cubic phases we see from Eq. (7) that the optimal value $f=0$ for the free-energy density is achieved for $w(\mathrm{v})=1 /[\mathrm{v} \Lambda(\mathrm{v})]$. For the spherical and cylindrical phases we find $w(\mathrm{~V})=3$ and $w(\mathrm{~V})=4$ from Eqs. (5) and (4). For each phase the corresponding line $w(\mathrm{v})$ lies in the middle of its region of stability; these lines are plotted in Fig. 6(a) instead of the full phase diagram for $r$ $=0$. In the following we will denote them as lines of vanishing frustration since they mark the specific parameter values for which a given phase can satisfy both bending and concentration constraints simultaneously. In the $(\mathrm{V}, w)$-plane, each of the structures considered has such a line, thus each of
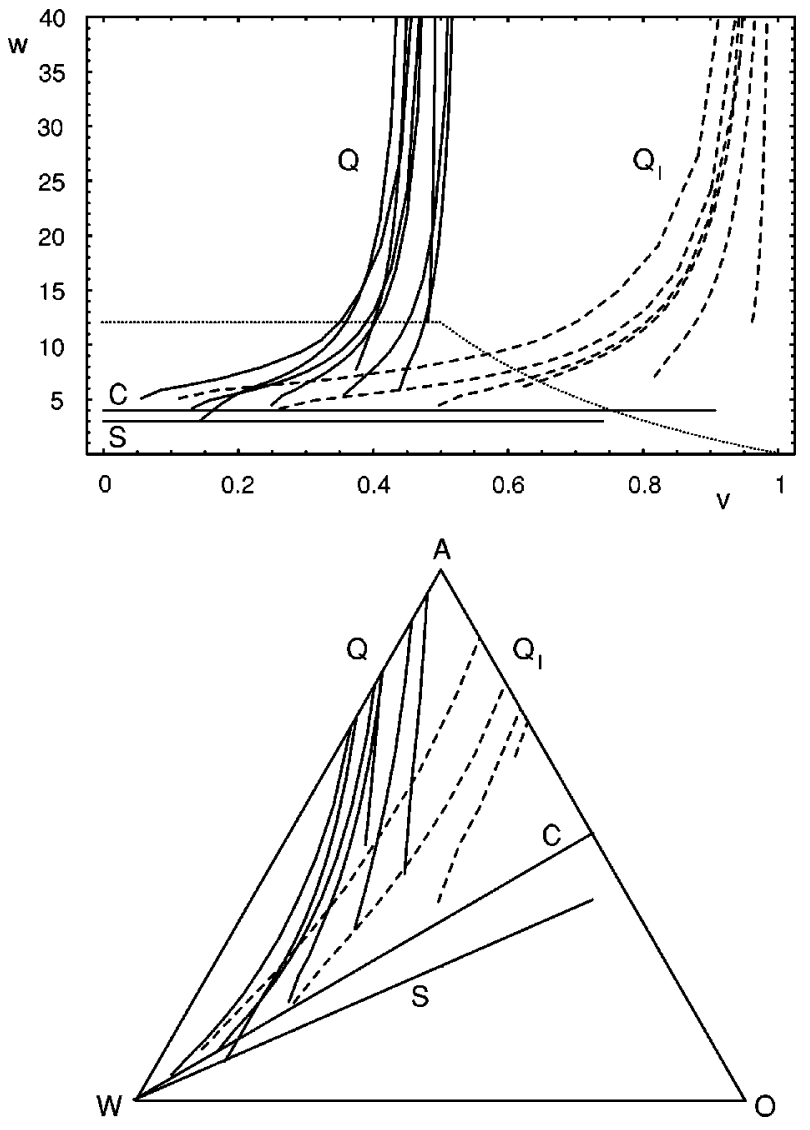

FIG. 6. Lines of vanishing frustration of various structures for $r=0$. In the $(\mathrm{V}, w)$-plane, the lines $w(\mathrm{~V})$ correspond to values for $(w, \mathrm{~V})$ where a specific phase has vanishing free-energy density. For $w \gg 1$ the $\mathrm{Q}$ and $\mathrm{Q}_{\mathrm{I}}$ structures are stable for $\mathbf{v} \leq \mathbf{v}_{0}$ and $\mathbf{v} \leq 1$, respectively. The lines at $w=3$ and $w=4$ correspond to spheres and cylinders, respectively. For $c_{0}=1 / 6$, only the part below the dotted line is mapped onto the Gibbs triangle.

them has some region of stability where its particular geometry serves best to accommodate the volume fractions of the different components. In fact there are even several values of $(w, \mathrm{~V})$ where the free-energy density of different structures is degenerate. In Fig. 6(b) we map the lines of vanishing frustration from Fig. 6(a) onto the Gibbs triangle for $c_{0}=1 / 6$. For these values, the $\mathrm{Q}$ structures run towards the W-A side and the $\mathrm{Q}_{\mathrm{I}}$ structures towards the $A-O$ side. Although the lines of vanishing frustration for $\mathrm{C}(\mathrm{P}), \mathrm{C}(\mathrm{P})_{\mathrm{I}}$, and $\mathrm{F}-\mathrm{RD}_{\mathrm{I}}$ are not mapped onto the Gibbs triangle, the phase behavior here remains highly degenerate.

Figure 6(a) demonstrates that the different structural types considered occupy different regions of the phase diagram in a very characteristic fashion; for large $w, \mathrm{Q}$, and $\mathrm{Q}_{\mathrm{I}}$ structures are stable for $\mathrm{v} \leqslant 1 / 2$ and $\mathrm{v} \leqslant 1$, respectively. With decreasing $w$, the regions of stability curve to the left. In order to understand the sequence of phases within the bandlike region of each structural type for large $w$, it is useful to expand the free-energy densities of the various bicontinuous structures about the minimal surface members by using Eq. (6). For the $\mathrm{Q}$ and $\mathrm{Q}_{\mathrm{I}}$ structures, this corresponds to an expansion about the volume fractions $\mathrm{V}_{0}$ and 1 , respectively. Again we only consider the lines of vanishing frustration for which $f=0$ and $w(\mathrm{v})=1 /[\mathrm{v} \Lambda(\mathrm{v})]$. The curvature index $\Lambda(\mathrm{v})$ defined in Eq. (8) can be approximated by 

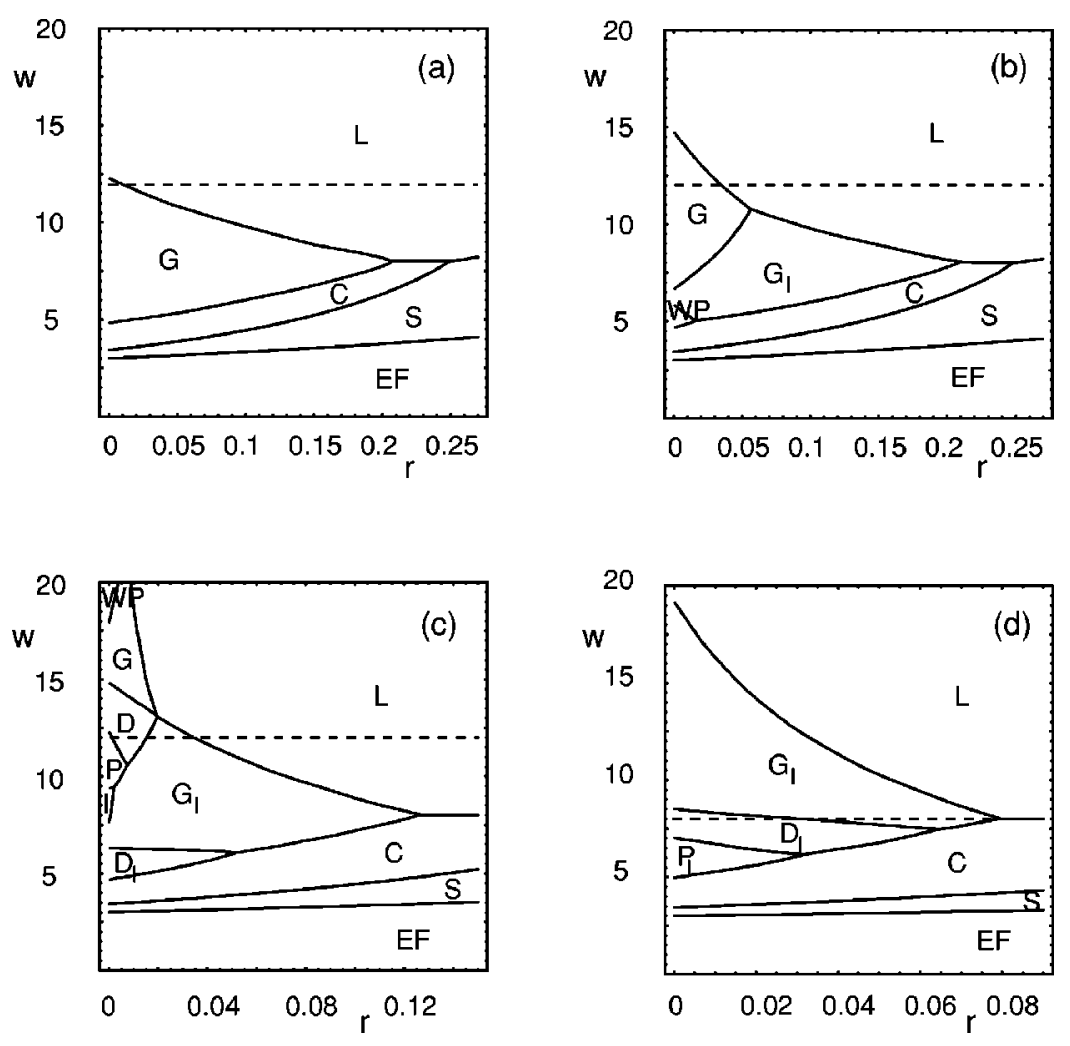

FIG. 7. Phase diagram as a function of $w$ and $r$ for (a) $\mathbf{v}=0.1$, (b) $\mathbf{v}=0.2$, (c) $\mathbf{v}=0.4$, and (d) $\mathbf{v}=0.6$. For $c_{0}$ $=1 / 6$, only the region below the dashed line is mapped onto the Gibbs triangle. The most stable phase with respect to $r$ is the double gyroid $\mathrm{G}_{\mathrm{I}}$ which however cannot exist for $\mathrm{v}<0.112$.

$$
\begin{aligned}
& \Lambda(\mathrm{v})=\frac{-\left(\mathrm{v}-\mathrm{v}_{0}\right)}{c A_{0}}+\mathcal{O}\left(\left(\mathrm{v}-\mathrm{v}_{0}\right)^{2}\right), \\
& \Lambda_{I}(\mathrm{v})=\frac{(1-\mathrm{v})}{4 c A_{0}}+\mathcal{O}\left((1-\mathrm{v})^{2}\right) .
\end{aligned}
$$

In the same order of approximation, the lines of vanishing frustration then follow as $w=-c A_{0} / \mathrm{v}_{0}\left(\mathrm{v}-\mathrm{v}_{0}\right)$ and $w=$ $-4 c A_{0} /(\mathrm{V}-1)$ for $\mathrm{Q}$ and $\mathrm{Q}_{\mathrm{I}}$ structures, respectively. Thus the minimal surface case corresponds to the stable solutions for $w \gg 1$ at $\mathbf{v}=\mathrm{v}_{0}$ and $\mathbf{v}=1$, respectively, where the curvature indexes of the corresponding structures disappear (compare Fig. 5). For the balanced single structures and the double structures the hierarchy of the different phases within the bandlike region occupied by a certain structural type is thus determined by the values of $c A_{0}$, which are given in Table I for the six families considered. The only exception are the four nonbalanced single structures, which cannot be compared in this way, since the value for $\mathrm{v}_{0}$ is different for each of them. Using the approximation $c \approx-A_{0}^{2} / 2 \pi \chi$ derived above, we find $c A_{0} \approx \Gamma_{0}^{2}$, so that the sequence is approximately determined by the topology index of the minimal-surface member of each family. In fact the structures in Table I are ordered with decreasing $\Gamma_{0}$. In particular, for a given structural type and values of the hydrocarbon volume $v$ outside the (rather restricted) $v$-intervals of existence of I-WP, F-RD, and C(P), we expect from Eq. (9) to find the sequence $\mathrm{G}-\mathrm{D}-\mathrm{P}$ as a function of either $\mathrm{V}$ or $w$.

\section{B. Phase diagrams for $r>0$}

When the saddle-splay modulus becomes negative, so that $r>0$, the free-energy densities of lamellae and cylinders do not change since these structures have vanishing Gaussian curvature. However, spheres have positive Gaussian curvature and therefore their free-energy density decreases [compare Eq. (5)]. Since bicontinuous cubic phases have negative (integral) Gaussian curvature, their free-energy density increases [compare Eq. (7)] and they will be suppressed towards large $w$ by the lamellar phase and towards small $w$ by the cylindrical phase; these phases in turn will for sufficiently large $r$ be suppressed by the spherical phase. Since ternary amphiphilic systems presumably have negative but small values of the saddle-splay modulus, the important questions here are up to which value of $r$ the bicontinuous cubic phases remain stable, and which of the 14 different structures considered performs best. It follows from Eq. (7) that the relevant quantity is the topology index: the larger its value for a certain bicontinuous cubic structure which is stable for $r=0$, the longer this structure stays stable with increasing $r$. Figure 5 shows that for any value of $\mathrm{v}$, the double structures have larger geometry indices than single structures. Within each of the two relevant structural classes, it is the gyroid structure which has the largest value of the topology index (compare Table I). We therefore conclude that the double gyroid $\mathrm{G}_{\mathrm{I}}$ should dominate phase behavior for $r>0$ for topological reasons. However, there are three restrictions to this general conclusion. First, $\mathrm{G}_{\mathrm{I}}$ can only realize $\mathrm{V} \in[0.112,1.0]$. Second, before $\mathrm{G}_{\mathrm{I}}$ can dominate all other bicontinuous cubic phases with increasing $r$, it might be already dominated itself by the lamellar phase $\mathrm{L}$ and the cylindrical phase C. And third, since the topological term is weighted by a factor $(w \mathrm{v})^{3}$ in Eq. (7), the double gyroid cannot perform so well for small $\mathrm{v}$ as it can for larger $\mathrm{v}$.

Our numerical results nicely corroborate this analysis and shows the exact outcome of the balance between the different principles mentioned. In Fig. 7 we show phase dia- 

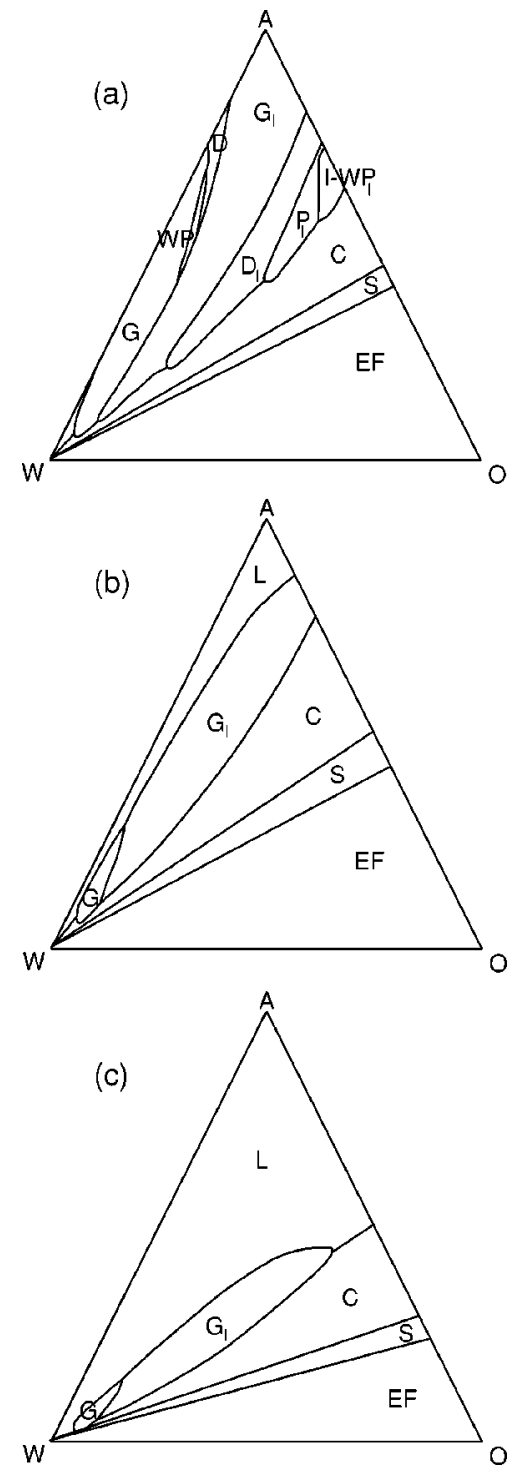

FIG. 8. Phase behavior in the Gibbs triangle for (a) $r=0.01, c_{0}=1 / 6$, (b) $r=1 / 15=0.067, c_{0}=1 / 6$, and (c) $r=1 / 15, c_{0}=1 / 12$. For larger values of $r$, the gyroid structures $G$ and $G_{I}$ dominate. Lowering spontaneous curvature $c_{0}$ corresponds to raising temperature and extends the region of stability for the lamellar phase L. For simplicity, in these plots we do not consider the close-packing constraint for $\mathrm{S}$.

grams as a function of $w$ and $r$ for $\mathbf{v}=0.1,0.2,0.4$, and 0.6. Since $w, \mathrm{v}$, and $r$ define the parameter space, these figures show nearly the complete phase behavior predicted by the model. Only the subsequent mapping onto the Gibbs triangle is affected by the chosen values for $\alpha$ and $c_{0}$. In Fig. 7, we draw the lines of constant $w$, which marks the upper edge of the part of the $(r, w)$-plane which is mapped onto the Gibbs triangle for $\alpha=1 / 2$ and $c_{0}=1 / 6$. The degeneracy of the bicontinuous cubic phases for $r=0$ discussed above disappears quickly with increasing $r$. They eventually all disappear because the lamellar phase $\mathrm{L}$ and the cylindrical phase $\mathrm{C}$ become more stable. At $r=0.25, \mathrm{C}$ itself is suppressed by the spherical phase $\mathrm{S}$. The double gyroid $\mathrm{G}_{\mathrm{I}}$ remains stable for larger values of $r$ than all other bicontinuous cubic phases. In fact for $\mathbf{V}=0.2$ [Fig. 7(b)] it is stable up to $r=0.2$. This result stands in marked contrast to the results of Ref. 10, which predicted that the most stable phase should be $D_{I}$ with a stability limit of $r=0.1$. For $\mathbf{V}=0.1$ [Fig. 7(a)] the double gyroid cannot exist and the single gyroid $\mathrm{G}$ is the only stable bicontinuous cubic phase. For $\mathrm{V}=0.6[$ Fig. $7(\mathrm{~d})]$ the single structures are not stable since their curvature index is negative for $\mathbf{v}>\mathrm{v}_{0}$ [compare Fig. 6(a)]. If more than one structure is stable within one structural class, we see the sequence G-D-P which was shown above to be determined by the topology index as well.

In Fig. 8 we show the Gibbs triangles for $r=0.01$ and $c_{0}=1 / 6, r=1 / 15=0.07$ and $c_{0}=1 / 6$, as well as $r=1 / 15$ and $c_{0}=1 / 12$. In the first case of a very small value of the saddlesplay modulus, one still sees the degeneracy of the case $r$ $=0$. For the more negative value, only the single gyroid $\mathrm{G}$ and the double gyroid $\mathrm{G}_{\mathrm{I}}$ are stable. Comparing with Fig. 6, we see that the G-phase is stable near its extremal volume fraction of $v=0.056$, while the $G_{I}$-phase is stable for a large range of volume fractions, which covers the region of the Gibbs triangle where its line of vanishing frustration is located for $r=0$. The effect of decreasing spontaneous curvature from $c_{0}=1 / 6$ in Fig. 8(b) to $c_{0}=1 / 12$ in Fig. 8(c) is to extend the region of stability of the lamellar and bicontinuous phases away from the $W-A$ side, towards the center of the Gibbs triangle.

\section{DISCUSSION AND CONCLUSIONS}

In order to calculate phase behavior of ternary amphiphilic systems, we investigated a simple curvature model with nonzero spontaneous curvature. In particular, we focused on the bicontinuous cubic phases whose interfaces were modeled by triply periodic surfaces of constant mean curvature. We showed that for this class of surfaces, the freeenergy density of the bicontinuous cubic phases can be written in a very general form which emphasizes the universal geometrical character of the problem studied. It consists of two terms; the bending term depends on the curvature index $\Lambda$ and the topological term depends on the topology index $\Gamma$. The relative strength of the two terms is determined by $r=$ $-\bar{\kappa} / 2 \kappa$. The relevance of the two quantities $\Lambda$ and $\Gamma$ not only depends on $r$, but also on the way by which they are weighted in the free-energy density by the two variables $w$ and $v$ which parametrize concentration space. Several properties have to conspire for a specific phase to be stable at a certain point of the phase diagram. First, its geometrical properties have to allow to accommodate the given concentrations (this imposes some constraints on the allowed values for the hydrocarbon volume fraction $v$ ). Second, the resulting mean curvature must be close to the given spontaneous curvature in order to keep the bending term small. Since there are two independent degrees of freedom in concentration space, one of which is sufficient to adjust the mean curvature to its optimal value, the regions of stability are at least one-dimensional in the $(\mathrm{V}, w)$-plane for $r=0$. The exact location of these lines of vanishing frustration is determined by the curvature index $\Lambda(\mathrm{v})$. Phases with negative curvature index $\Lambda$ are less stable than the lamellar phase. For positive spontaneous curvature (monolayer bending towards oil regions), this rules out the single structures for $\mathrm{v}>\mathrm{V}_{0}$ and the 
double structures of type II. Third, for $r>0$ the structure has to be favored by the topological term also. This requires large values for the topology index $\Gamma(\mathrm{v})$ and basically favors the double gyroid $\mathrm{G}_{\mathrm{I}}$. Fourth, the concentrations in the regions of stability have to be physically relevant, i.e., they have to correspond to those of the Gibbs triangle. The mapping of the phase diagram as a function of $v$ and $w$ onto the Gibbs triangle depends on specific values for the amphiphile chain length $\alpha$ and the spontaneous curvature $c_{0}$ and cuts off some of the phase behavior in the $(\mathrm{V}, w)$-plane.

The phase behavior for $r=0$ is highly degenerate. This degeneracy has been discussed already in Ref. 41 for the Canham-Helfrich Hamiltonian without spontaneous curvature and concentration constraints. In order to resolve the question of the relative stability of the different bicontinuous cubic phases, one has to consider further physical effects like topological contributions $(r>0)$, van der Waals, electrostatic or steric interactions, higher order curvature terms ${ }^{41}$ or packing energies for the hydrocarbon chains. ${ }^{42,43}$ Our analysis shows that although every phase considered has some region of stability in phase space, there are certain general principles which allow to understand the complicated structure of the resulting phase diagram. The locations of the regions of stability are determined by the curvature index $\Lambda$; we have derived a simple approximation for $\Lambda$, which is valid for large $w$ and explains why $\mathrm{Q}$ and $\mathrm{Q}^{\mathrm{I}}$ structures are stable for $\mathrm{v} \leq 1 / 2$ and $\mathrm{v} \leq 1$, respectively. Moreover, it turns out that the sequence of phases within the bandlike regions of a certain structural type is determined by the value of $c A_{0}$, which in turn can be well approximated by $\Gamma_{0}^{2}$. Thus the relative location of the different phases of one type is determined by the topology index of the minimal-surface member of that family.

When the saddle-splay modulus becomes negative, more and more of the cubic bicontinuous phases disappear until eventually all of them are suppressed by the noncubic phases. However, we found that the bicontinuous cubic phases remain stable for considerably higher values of $r$ than found previously. ${ }^{10}$ Since the structure performs best which has both a high topology index and can accommodate large ranges of hydrocarbon volume fraction $\mathrm{v}$, the double gyroid $\mathrm{G}_{\mathrm{I}}$ becomes the most stable bicontinuous cubic phase for increasing $r$. Note that $\mathrm{G}_{\mathrm{I}}$ is the only double structure of type I where the oil-filled labyrinths consist of channels, which are connected by junctions of threefold coordination exclusively. Its outstanding stability with respect to $r$ can therefore be explained by the fact that it is the most cylinderlike of the bicontinuous cubic phases. The same geometrical property in fact can stabilize also the entropy-dominated microemulsion since low-coordinated vertices provide a lot of configurational entropy. ${ }^{44}$ Since with increasing $r$ the double gyroid $\mathrm{G}_{\mathrm{I}}$ is eventually suppressed by the lamellar and the cylindrical phase before it can suppress all other bicontinuous cubic phases, the single gyroid $G$ (with has the most favorable topology index within its structural type) has a considerable region of stability as well.

In summary, we have demonstrated that the complicated phase behavior of bicontinuous cubic phases in ternary systems can be understood in terms of the interplay between their universal geometrical properties and the concentration constraints of a ternary system. The main result is that the gyroid structures are favored since they have the largest values for the topology index $\Gamma$, that is the smallest porosity among the bicontinuous cubic phases. It should be pointed out, however, that this work rests on the basic assumption that all structures form surfaces of constant mean curvature. Such surfaces arise as minima of $\int d A$ under a volume constraint, or as minima of $\int d A\left(H-c_{0}\right)^{2}$ without volume constraint, but they are not solutions to $\int d A\left(H-c_{0}\right)^{2}$ under volume constraint-except for the special case of vanishing frustration, where the surface with $H=c_{0}$ just satisfies the volume constraint. It is easy to understand that, in general, surfaces of constant mean curvature do not minimize the curvature energy with volume constraint, since the energy can be lowered by keeping the mean curvature on the largest part of the surface very close to $c_{0}$, and by concentrating deviations from $H=c_{0}$-which are enforced by the volume constraint - to a small part of the surface (which therefore makes a small contribution to the curvature integral). However, since the regions of stability of most bicontinuous phases do not extend very far from the lines of vanishing frustration in the phase diagram, the deviations of the exact solutions from surfaces of constant mean curvature can be expected to be small for the physically relevant regions.

In this work, we focused on the case of finite and positive spontaneous curvature. Its value can be controlled in amphiphilic systems by changing temperature and disappears at the balanced temperature. Above the balanced temperature, the spontaneous curvature is negative and oil and water have to be interchanged in the structures and phase diagrams discussed. Then the double structures of type I are replaced by the double structures of type II (the inverse phases) and the phase boundaries in the Gibbs triangle run predominantly towards the oil apex. For surfactant systems, the balanced temperature is usually well above room temperature. ${ }^{17} \mathrm{Al}$ though the curvature model presented here predicts inverse bicontinuous phases above the balanced temperature, they are probably destroyed by thermal fluctuations in this case. For lipid systems, the balanced temperature is usually similar to the main transition temperature, and inverse phases are predicted even at room temperature. In accordance with experiments on both kinds of systems, the model presented in this paper predicts that the lamellar phase dominates at the balanced temperature. However, since it assumes finite spontaneous curvature and neglects thermal fluctuations, it cannot describe the bicontinuous microemulsion phase which for surfactant systems often coexists with the lamellar phase around the balanced temperature.

We did not consider the effect of thermal fluctuations or long-ranged interactions. The special case of vanishing spontaneous curvature has been investigated in Ref. 41. It was found that for the Canham-Helfrich model, the elastic bulk and shear moduli vanish at $T=0$. Small higher order curvature terms make these moduli finite, but thermal longwavelength fluctuations with large amplitudes should remain. An extension of this type of analysis to systems with spontaneous curvature has not been attempted so far. Since the spontaneous curvature introduces a new length scale, 
many of the results of Ref. 41 should not apply in this case. However, large fluctuations on long length scales can still be expected. The contribution of the fluctuations to the freeenergy density should be of the form $k_{B} T \ln (\delta / a) / a^{3}$, where $a$ is the lattice constant, which is determined by the concentrations, and $\delta$ is a molecular length of the amphiphile. ${ }^{41,45,46}$ Therefore, the thermal contributions to the free energy of different bicontinuous cubic phases should be very similar, and the sequences of stable bicontinuous cubic phases as a function of concentrations-as shown in Fig. 8 and explained in terms of their geometrical properties in Sec. IVshould be observed in experiments. In fact for the system DDAB-water-styrene a sequence of double structures was reported which seems to correspond to increasing topology index. ${ }^{33}$ For the noncubic phases, thermal fluctuations correspond mainly to steric interactions between fluctuating lamellae and cylinders and translational entropy for spheres. Extending the model by these contributions would in fact remove the nonconcavity of their free energy densities. Previous work suggests that the main effect for surfactant systems would be to favor micellar phases near the wateramphiphile side of the phase diagram and lamellar phases near the water-apex. ${ }^{47}$ Such a modification could bring the calculated phase diagrams of Fig. 8 in good qualitative agreement with experimental phase diagrams measured for the whole Gibbs triangle, like the ones obtained in Ref. 32 for $\mathrm{H}_{2} \mathrm{O} / \mathrm{C}_{10} / \mathrm{C}_{12} \mathrm{E}_{5}$. For a more detailed comparison, experimental structure determination for bicontinuous cubic phases in ternary surfactant systems is needed. The same holds true for ternary lipid systems where to our knowledge hardly any experimental data are known.

With respect to long-ranged interactions, it seems reasonable to assume similar effects for all bicontinuous cubic phases. For attractive/repulsive forces, we therefore expect all of them to be favored/disfavored to a similar degree. In principle such additional contributions could stabilize the bicontinuous cubic phases. In regard to the noncubic phases, it was shown previously that van der Waals interactions should show a considerable effect only for large values of the bending rigidity, i.e., for lipids. ${ }^{47}$ Thus we predict that the geometrical arguments presented in this work describe the main physics of bicontinuous cubic phases in ternary amphiphilic systems with spontaneous curvature, even when more complicated models are considered.

\section{ACKNOWLEDGMENTS}

We thank K. Große-Brauckmann for helpful discussions and J. Schmalzing and H. Wagner for drawing our attention to the connection to integral geometry. U.S.S. gratefully acknowledges support by the Minerva Foundation.

\footnotetext{
${ }^{1}$ Micelles, Membranes, Microemulsions, and Monolayers, edited by W. M. Gelbart, A. Ben-Shaul, and D. Roux (Springer, New York, 1994).

${ }^{2}$ Structure and Dynamics of Membranes, Vols. 1A and 1B in Handbook of Biological Physics, edited by R. Lipowsky and E. Sackmann (Elsevier, Amsterdam, 1995).

${ }^{3}$ K. Fontell, Colloid Polym. Sci. 268, 264 (1990).
}

${ }^{4}$ V. Luzzati et al., J. Mol. Biol. 229, 540 (1993).

${ }^{5}$ J. M. Seddon and R. H. Templer, in Structure and Dynamics of Membranes: From Cells to Vesicles, Vol. 1A in Handbook of Biological Phys$i c s$, edited by R. Lipowsky and E. Sackmann (Elsevier, Amsterdam, 1995), pp. 97-160.

${ }^{6}$ International Workshop on Geometry and Interfaces at Aussois, France, Vol. C7 in J. Phys. (Paris) Colloq, edited by E. Dubois-Violette and B. Pansu (Les Editions de Physique, Les Ulis Cedex A, 1990).

${ }^{7}$ J. Klinowski and A. L. Mackay, eds, Curved Surfaces in Chemical Structure, Vol. 354 in Philosophical Transactions of the Societies of London, A (The Royal Society, London, 1996).

${ }^{8}$ S. A. Safran and L. A. Turkevich, Phys. Rev. Lett. 50, 1930 (1983).

${ }^{9}$ S. A. Safran, L. A. Turkevich, and P. Pincus, J. Phys. (France) Lett. 45, L69 (1984).

${ }^{10}$ Z.-G. Wang and S. A. Safran, Europhys. Lett. 11, 425 (1990).

${ }^{11}$ D. M. Anderson, Ph.D. thesis, University of Minnesota, 1986.

${ }^{12}$ D. M. Anderson, H. T. Davis, L. E. Scriven, and J. C. C. Nitsche, Adv. Chem. Phys. 77, 337 (1990).

${ }^{13}$ K. Große-Brauckmann, J. Colloid Interface Sci. 187, 418 (1997).

${ }^{14}$ K. Große-Brauckmann, Exp. Math. 6, 21 (1997).

${ }^{15}$ P. B. Canham, J. Theor. Biol. 26, 61 (1970).

${ }^{16}$ W. Helfrich, Z. Naturforsch. C 28, 693 (1973).

${ }^{17}$ R. Strey, Colloid Polym. Sci. 272, 1005 (1994).

${ }^{18}$ W. Helfrich, in Physics of Defects, Les Houches Summer School 1980, edited by R. Balian, M. Kleman, and J.-P. Poirier (North-Holland, Amsterdam, 1981).

${ }^{19}$ J. C. C. Nitsche, Lectures on Minimal Surfaces (Cambridge University Press, Cambridge, 1989).

${ }^{20}$ U. Dierkes, S. Hildebrandt, A. Küster, and O. Wohlrab, Minimal Surfaces I and II, Vol. 295 in Grundlehren der Mathematischen Wissenschaften (Springer, Berlin, 1992).

${ }^{21}$ A. H. Schoen, NATO Technical Report No. D-5541, Washington, D.C., 1970.

${ }^{22}$ H. Karcher and K. Polthier, Philos. Trans. R. Soc. London, Ser. A 354, 2077 (1996).

${ }^{23}$ W. Fischer and E. Koch, Philos. Trans. R. Soc. London, Ser. A 354, 2105 (1996).

${ }^{24}$ H. Karcher, Manuscr. Math. 64, 291 (1989).

${ }^{25}$ D. M. Anderson and H. Wennerström, J. Phys. Chem. 94, 8683 (1990).

${ }^{26}$ M. Spivak, A Comprehensive Introduction to Differential Geometry I-IV (Publish or Perish, Houston, 1979).

${ }^{27}$ S. T. Hyde, J. Phys. Chem. 93, 1458 (1989).

${ }^{28}$ V. Luzzati et al., Nature (London) 220, 485 (1968).

${ }^{29}$ G. Gompper and S. Klein, J. Phys. II 2, 1725 (1992).

${ }^{30}$ G. Gompper and U. S. Schwarz, Z. Phys. B: Condens. Matter 97, 233 (1995).

${ }^{31}$ A. Linhananta and D. E. Sullivan, Phys. Rev. E 57, 4547 (1998).

${ }^{32}$ M. S. Leaver et al., J. Chem. Soc., Faraday Trans. 91, 4269 (1995).

${ }^{33}$ P. Ström and D. M. Anderson, Langmuir 8, 691 (1992).

${ }^{34}$ J. O. Räler, S. Radiman, A. de Vallera, and C. Toprakcioglu, Physica B 156, 398 (1989).

${ }^{35}$ P. Mariani, V. Luzzati, and H. Delacroix, J. Mol. Biol. 204, 165 (1988).

${ }^{36}$ U. S. Schwarz and G. Gompper, Phys. Rev. E 59, 5528 (1999).

${ }^{37}$ R. Schneider, Convex Bodies: The Brunn-Minkowski Theory (Cambridge University Press, Cambridge, 1993).

${ }^{38}$ A. Fodgen and S. T. Hyde, Eur. Phys. J. B 7, 91 (1999).

${ }^{39}$ M. Teubner, Europhys. Lett. 14, 403 (1991).

${ }^{40}$ G. Gompper and J. Goos, Phys. Rev. E 50, 1325 (1995)

${ }^{41}$ R. Bruinsma, J. Phys. II 2, 425 (1992).

${ }^{42}$ D. M. Anderson, S. M. Gruner, and S. Leibler, Proc. Natl. Acad. Sci. USA 85, 5364 (1988).

${ }^{43}$ P. M. Düsing, R. H. Templer, and J. M. Seddon, Langmuir 13, 351 (1997).

${ }^{44}$ T. Tlusty, S. A. Safran, R. Menes, and R. Strey, Phys. Rev. Lett. 78, 2616 (1997).

${ }^{45}$ G. Porte, J. Phys.: Condens. Matter 4, 8649 (1992).

${ }^{46}$ D. C. Morse, Phys. Rev. E 50, R2423 (1994).

${ }^{47}$ U. S. Schwarz, K. Swamy, and G. Gompper, Europhys. Lett. 36, 117 (1996).

${ }^{48}$ D. Cvijovic and J. Klinowski, Chem. Phys. Lett. 226, 93 (1994). 